Final Report for

Grant \# DE-FG02-04ER46137

\title{
Mechanisms of High Temperature/Low Stress Creep of Ni-Based Superalloy Single Crystals
}

\author{
Submitted to the U. S. Department of Energy
}

Basic Energy Sciences Program

by

Professor Michael J. Mills

mills.108@osu.edu

Department of Materials Science and Engineering

The Ohio State University

Columbus, $\mathrm{OH} 43210$

5 March 2009 


\section{INTRODUCTION}

Cast nickel-based superalloys are used for blades in land-based, energy conversion and powerplant applications, as well as in aircraft gas turbines operating at temperatures up to $1100^{\circ} \mathrm{C}$, where creep is one of the life-limiting factors. Creep of superalloy single crystals has been extensively studied over the last several decades. Surprisingly, only recently has work focused specifically on the dislocation mechanisms that govern high temperature and low stress creep [1-3]. Nevertheless, the perpetual goal of better engine efficiency demands that the creep mechanisms operative in this regime be fully understood in order to develop alloys and microstructures with improved high temperature capability. At present, the micro-mechanisms controlling creep before and after rafting [4] (the microstructure evolution typical of high temperature creep) has occurred have yet to be identified and modeled, particularly for [001] oriented single crystals. This crystal orientation is most interesting technologically since it exhibits the highest creep strength.

The major goal of the program entitled "Mechanisms of High Temperature/Low Stress Creep of Ni-Based Superalloy Single Crystals" (DOE Grant \# DE-FG0204ER46137) has been to elucidate these creep mechanisms in cast nickel-based superalloys. We have utilized a combination of detailed microstructure and dislocation substructure analysis combined with the development of a novel phase-field model for microstructure evolution. 


\section{EXPERIMENTAL RESULTS}

The focus of our experimental studies has been TEM analysis of two diferent single crystal alloys following creep. The NASAIR alloy is a "first-generation" alloy while Rene N5 is a compositionally more complex, "third generation" alloy. In spite of exhaustive mechanical testing of these alloys, little or no substructure analysis is available regarding the deformation mechanisms of this alloy in the open literature. A series of creep single crystal N5 tensile creep specimens were analyzed in terms of phase evolution and dislocation substructure variation as a function of creep strain. A postdoctoral research associate, Dr. Peter Sarosi, and graduate research assistant, Clarissa Yablinsky, has been supported by this program in this effort. We have focused on examination of longitudinal (instead of transverse) specimen sections in order to more readily characterize phase morphology and dislocation structures. Deformation substructures have been examined using bright field STEM imaging of dislocations. Although not a new technique [5-6] it has not been widely utilized for deformation substructure analysis. However, we have found it to be particularly useful for examining dislocation structures in fine-grained polycrystalline materials, and for obtaining large fields of view for assaying deformation mechanisms and dislocation density measurements over relatively large specimen regions. Since this technique produces images while sampling a range of diffraction angles, dislocations produce contrast even when specimens are bent and have varying thickness - effects which generally obscure dislocations when using conventional diffraction contrast imaging.

In both alloys, the rafting process is observed to be complete after only $1 \%$ strain. As a consequence, the f.c.c. $\gamma$ phase and $\mathrm{L}_{2} \gamma^{\prime}$ phase form an irregular, alternating 
structure. The layers are aligned perpendicular to the applied tensile axis, as expected based on previous studies of negative lattice misfit alloys [7-8]. The deformation mechanisms observed in the NASAIR alloy at a single strain level have been documented in a recent publication [9]. Below we briefly present additional results for the Rene N5 alloy that provide further insight into the evolution of the microstructure and substructure with strain. These results are being submitted for publication [10]. A representative creep curve and examples of BF-STEM analysis of dislocation structures following $1.25 \%$ and $6.3 \%$ creep strain are shown in Figure 1. Each layer is relatively continuous after small creep strains. Extensive dislocation activity is apparent in the $\gamma^{\prime}$ phase in all samples examined. Dislocations can be observed traversing the $\gamma^{\prime}$ phase in numerous locations. These dislocations are clearly connected to the interfacial networks at the $\gamma / \gamma^{\prime}$ interfaces on either side of the $\gamma^{\prime}$ phase, and are predominantly $a[100]$ and $a[010]$ type, with a smaller number of $\mathrm{a}[001]$ and $\mathrm{a}<110>$ type dislocations. The presence of the $a[100]$ and $a[010]$ dislocations, as exemplified in Figure 1(b), is remarkable since there is no resolved glide or climb force operating on them due to the applied stress. These observations support a qualitative model for creep of rafted structures which assumes that the channeling of the $a<100>$ dislocations through the $\gamma^{\prime}$ phase is the rate-limiting process. There also exist dislocations traversing the $\gamma$ phase as well. In our model, motion of these matrix dislocations actually produce work due to the applied stress, unlike the channeling $a[100]$ and $a[010]$ dislocations in the $\gamma^{\prime}$ phase. The critical role of the latter, however, is to eliminate interface dislocation content, thereby reducing the local back stress such that matrix dislocation activity can proceed. As the $\gamma$ phase becomes discontinuous at larger strains, then the dislocations in the $\gamma^{\prime}$ are no longer 
constrained to channel laterally, but may also traverse to neighboring $\gamma^{\prime}$ layers, thereby dramatically increasing the effect that each individual $\gamma^{\prime}$ dislocation can have on the recovery of the interfacial networks in many layers. In addition, the density of dislocations having a Burgers vector parallel to the applied stress direction increases with strain (dislocations indicated in blue in Figure 1(c)). Thus, these observations also provide important insight into the origin of the gradually increasing creep rate (see Figure 1(a)), typically observed in tension during high temperature creep of blade alloys.
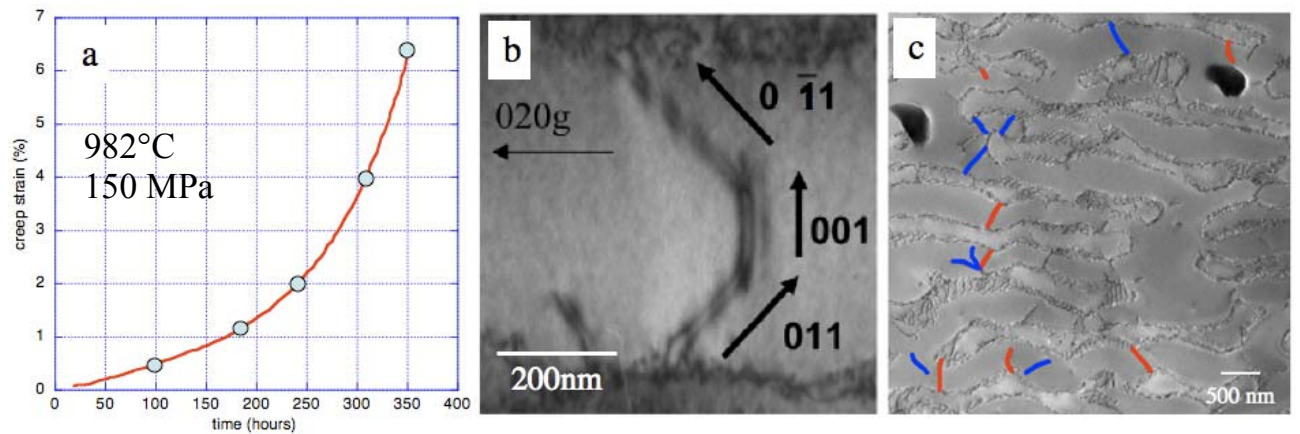

Figure 1: (a) Representative creep curve for Rene N5 indicating strain levels at which TEM analysis has been performed, (b) TEM image of an a $<100>$ dislocation threading across a $\gamma^{\prime}$ raft at $1.25 \%$ strain, and (c) BF-STEM image indicating a $<100>$ type (red) versus a $<011>$ type (blue) dislocations in the $\gamma^{\prime}$ after $6.3 \%$ strain. Note also the vertical contiguity of the $\gamma^{\prime}$ phase.

\section{PHASE FIELD MODELING RESULTS}

A ground-breaking phase field model that reproduces these remarkable morphological changes has also been developed under this program in collaboration with Prof. Yunzhi Wang and his graduate student, Ning Zhou, and initial results of this model 
are described below and in [11]. In this model, dislocation motion in $\gamma$ channels is driven by the local stress. This local stress includes contributions from lattice misfit, the external load, and misfit dislocations at the $\gamma / \gamma^{\prime}$ interface. Simultaneously, the diffusionmediated evolution of microstructure is determined by both chemical driving forces and elastic interactions with dislocations, as well as the effect of lattice misfit. Through this integrated approach, rafting of the $\gamma^{\prime}$ phase has been predicted under various conditions of misfit sign and loading type, and the results agree with our experiments and other analyses. It has also been found that modulus mismatch is not a necessary condition for rafting, as has been assumed previously in the elasticity-based models for rafting [12-13].

Under the homogeneous modulus assumption (ignoring the modulus mismatch between $\gamma$ and $\gamma^{\prime}$ phase), the simulation results show rafting of $\gamma^{\prime}$ precipitates is closely related to the type and distribution of dislocations at the $\gamma / \gamma^{\prime}$ interfaces. The sign of lattice misfit and the direction of the applied load determine the dislocation configurations in the $\gamma$-channels and the rafting directions. It is found that dislocations prefer normal channels (normal to the direction of applied load) for a positive misfit alloy under compression, and a negative misfit alloy under tension which later develop $\mathrm{N}$ type rafting, while parallel channels were preferred by dislocations in a positive misfit alloy under tension and a negative misfit alloy under compression which develop P type rafting.

A phase field model that captures the spatial-temporal evolution of precipitate microstructure under elastic inhomogeneity (the elastic stiffness constants are different for matrix and precipitate) for Ni-base blade alloys has also been developed [14]. Simulation parameters such as lattice misfit, elastic moduli and the magnitude of applied stress have been carefully selected to match real experimental conditions due to the 
quantitative nature of the study. Simulation results show that when ignoring channel plasticity, the direction of rafting depends on the sign of the following products: $\frac{\sigma^{e x}\left[\left(C_{11}-C_{12}\right)_{\gamma^{\prime}}-\left(C_{11}-C_{12}\right)_{\gamma}\right]}{\delta\left[\left(C_{11}-C_{12}\right)_{\gamma}\right]^{2}}$. Where $\sigma^{e x}$ being applied stress and $\delta$ the lattice misfit. $\mathrm{C}_{11}, \mathrm{C}_{12}$ are the stiffness constants. It is found that rafting caused purely by modulus mismatch takes much longer time to complete compared with the simulations described above considering only channel plasticity. Thus, these simulations have for the first time clarified the relative importance of these effects on the rafting process.

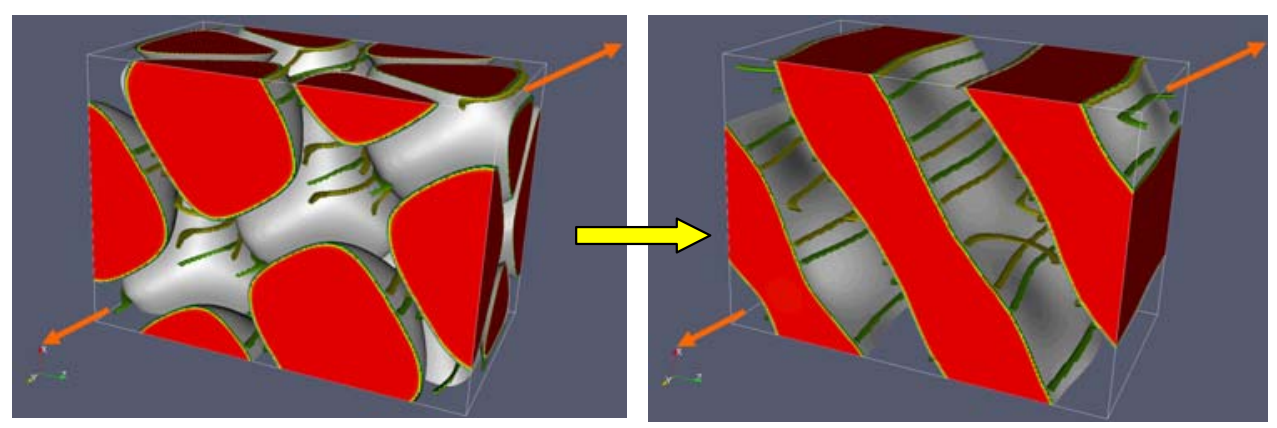

Figure 2: Phase field model result showing $N$ type rafting for alloys with $-0.3 \%$ lattice misfit under $152 \mathrm{MPa}$ tensile stress along [001] after 11 hours. 


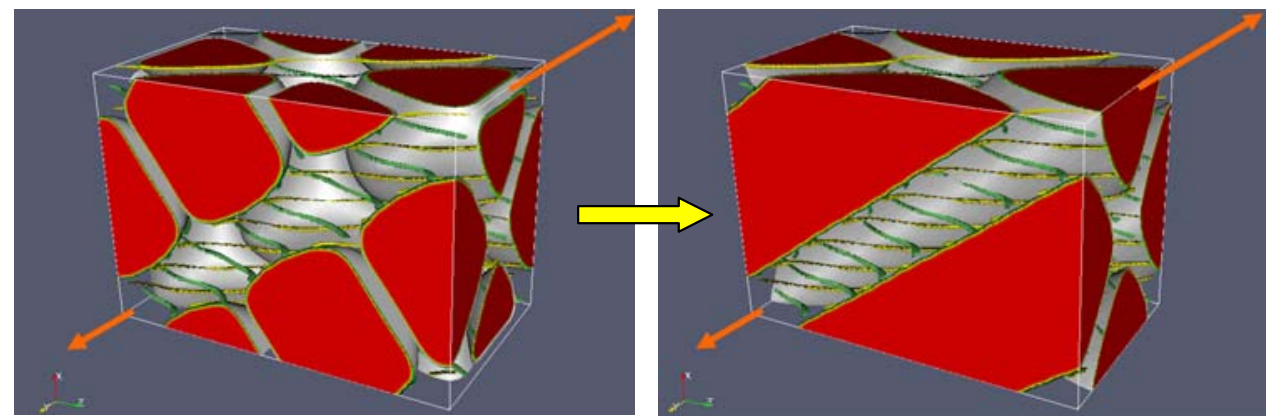

Figure 3: Simulation for $\mathrm{P}$ type rafting for alloys with $+0.3 \%$ lattice misfit under $152 \mathrm{MPa}$ tensile stress along [001] after 7 hours.

Finally, using an effective transformation strain to describe the channel plasticity, a simplified phase field model is being developed to take into account both channel dislocations and modulus mismatch. Preliminary test results show that the channel dislocations are the dominant factor determining the rafting mode $\left(\gamma^{\prime}\right.$ coarsening direction), while modulus difference between $\gamma / \gamma^{\prime}$ will slightly modify the kinetics of rafting process. Specifically, when $\gamma^{\prime}$ precipitate is "softer" than $\gamma$ matrix, rafting will take longer time for the same conditions. Thus, this model is enabling for the first time a quantitative evaluation of the relative importance of elastic misfit versus channel dislocation content to the rafting process. 


\section{PERSONNEL SUPPORTED}

- Peter Sarosi was a post-doctoral research associate who has been conducting advanced TEM investigation of the superalloys.

- Clarissa Yablinsky is a graduate research associate who has been developing our FIB-based capabilities for TEM sample preparation, and has also been conducting TEM investigation of the substructure of single crystal superalloys.

- Ning Zhou was a graduate research associate who has been developing the phase field modeling capability for treating rafting and creep in the high-misfit, Ni-base superalloys.

\section{REFERENCES}

(1) T. M. Pollock and A. S. Argon, Acta metall. mater., 40, 1 (1992).

(2) G. Eggeler and A. Dlouhy, Acta mater., 45, 4251(1997).

(3) R. Srinivasan, G. F. Eggeler and M. J. Mills, Acta mater., 48, 4867 (2000).

(4) J. K. Tien and S. M. Copley, Metall. Trans., 2, 215 (1971).

(5) C. J. Humphreys, Ultramicroscopy, 7, 7 (1981).

(6) D. M Maher, and D. C. Joy, Ultramicroscopy, 1, 239 (1976).

(7) M. V. Nathal and L. J. Ebert, Metall. Trans. A, 16A, 427 (1985).

(8) A. Epishin, T. Link, U. Bruckner and P. D. Portella, Acta Mater., 49, 4017 (2001).

(9) P. Sarosi, R. Srinivasan, M. V. Nathal, G. F. Eggeler and M. J. Mills, Acta mater., 55, 2509-2518 (2007).

(10) P. Sarosi, C. Yablinsky and M. J. Mills, Scripta mater., (2007), submitted for publication.

(11) N. Zhou, C. Shen, M.J. Mills and Y. Wang, Acta mater., 55, 5369-5381 (2007).

(12) A. Pineau. Acta metall. mater. 24, 559 (1976).

(13) S. Socrate and D. M. Parks. Acta metall. mater., 41, 2185 (1993).

(14) N. Zhou, C. Shen, M. J. Mills and Y. Wang, Acta Mater, submitted for publication (2007). 


\section{DOE SPONSORED PUBLICATIONS}

1. N. Zhou, C. Shen, M. J. Mills and Y. Wang, "Phase Field Modeling of $\gamma^{\prime}$ Rafting in Single Crystal Ni-Al Alloys," Proceedings of the International Conference on Advanced Materials Design \& Development (ICAMMD), CD-ROM, edited by M. Chakraborty, S. Ghosh, C. Jacob, D. Bhattacharya, V. Srinivas and T. K. Nath (2005).

2. P. Sarosi, R. Srinivasan, M. V. Nathal, G. F. Eggeler and M. J. Mills, "Importance of $a<100>$ Dislocations During the High Temperature Creep of Ni Base Superalloys Single Crystals Deformed Along the [001] Orientation", Acta mater. 55, pp. 25092518 (2007).

3. N. Zhou, C. Shen, M. J. Mills and Y. Wang, "Phase Field Modeling of Channel Dislocation Activity and $\gamma^{\prime}$ Rafting in Single Crystal Ni-Al," Acta mater., 55, pp. 5369-5381 (2007).

4. N. Zhou, C. Shen, P.M. Sarosi, M.J. Mills, T.M. Pollock and Y. Wang, " $\gamma$ ' Rafting in Single Crystal Blade Alloys - A Simulation Study,” Mat. Sci. Tech. (in press Feb. Formatted: Font: Times, $12 \mathrm{pt}$ Not Bold, Font color: Auto Formatted: Normal, Indent: Left: $0 \mathrm{pt}$, Hanging: $18 \mathrm{pt}$, Line spacing: Double issue, 2009).

5. N. Zhou, C. Shen, M. J. Mills and Y. Wang, "Contributions from Elastic Inhomogeneity and from Plasticity to $\gamma^{\prime}$ Rafting in Single Crystal Ni-Al," Acta Mater. 56 (2008) 6156-6173. 